\title{
Curable Resection in Gastric and Lymph Node Metastases From Melanoma
}

\author{
Yasunori Otowa, MD, Satoshi Suzuki, MD, PhD, Tatsuya Imanishi, MD, PhD, \\ Tetsu Nakamura, MD, PhD, Kenichi Tanaka, MD, PhD, Masanobu Sakaguchi, MD, PhD, \\ Toshinori Bito, MD, PhD, Yoshihiro Kakeji, MD, PhD \\ Department of Surgery, Kobe University Graduate School of Medicine, Kobe, Japan (Drs. Otowa, Suzuki, Imanishi, \\ Nakamura, Tanaka, and Kakeji). \\ Department of Internal Related, Kobe University Graduate School of Medicine, Kobe, Japan (Drs. Sakaguchi and Bito).
}

\begin{abstract}
We herein report a rare case of gastric and regional lymph node metastasis of cutaneous malignant melanoma that underwent curative resection. The patient, a 68-year-old man, was first diagnosed as having cutaneous malignant melanoma of the right forearm in 2005. He had extensive skin excision and axillary lymph node dissection and had undergone adjuvant chemotherapy. Six years after the primary surgery, gastrointestinal endoscopy revealed gastric metastasis of a malignant melanoma. As there was no other metastasis found, laparoscopic-assisted distal gastrectomy with lymph node dissection was performed. Microscopic findings showed diffuse melanin granule growth invading the muscularis propria of the stomach. Micrometastases of the lymph nodes were observed that were not detected by preoperative examination. Seventeen months have passed without recurrence. We conclude that regional lymph node dissection should be performed with gastrectomy whenever distant metastases are not observed, because there is a possibility of micrometastases, which cannot be detected preoperatively.
\end{abstract}

Key Words: Gastrectomy, Gastric metastasis, Laparoscopic surgery, Lymph node metastasis, Melanoma.

Citation Otowa Y, Suzuki S, Imanishi T, Nakamura T, Tanaka K, Sakaguchi M, Bito T, Kakeji Y. Curable resection in gastric and lymph node metastases from melanoma. CRSLS e2014.00310. DOI: 10.4293/CRSLS.2014.00310.

Copyright $(2014$ SLS This is an open-access article distributed under the terms of the Creative Commons Attribution-Noncommercial-ShareAlike 3.0 Unported license, which permits unrestricted noncommercial use, distribution, and reproduction in any medium, provided the original author and source are credited.

Address correspondence to: Yasunori Otowa, MD, Department of Surgery, Kobe University Graduate School of Medicine, 7-5-2 Kusunoki-chou, Chuo-ku, Kobe

650-0017, Japan. Telephone: +81-78-382-5925, Fax: +81-78-382-5939, E-mail: otoway@med.kobe-u.ac.jp

\section{INTRODUCTION}

Metastatic melanoma is a poor prognosis disease for which multiple metastases are often observed. Metastatic malignant melanoma of the gastrointestinal tract is said to be seen in $4 \%$ of patients. ${ }^{1}$ Autopsy data indicate that the small intestine was the most commonly involved metastatic site (58\%), followed by the stomach $(26 \%))^{2}$ However, most gastrointestinal metastasis are not discovered due to lack of digestive symptoms and mostly are accompanied with other multiple distant metastases.

We report a case with gastric and regional lymph node metastasis of malignant melanoma, which underwent curative resection by laparoscopic surgery. To our knowledge, this is the first case to report a curative resection of gastric metastasis and lymph node metastases of malignant melanoma.

\section{CASE REPORT}

A 68-year-old man was first diagnosed as having cutaneous malignant melanoma of the right forearm in 2005. He had extensive skin excision and axillary lymph node dissection. According to American Joint Committee on Cancer, ${ }^{3}$ the histology was T4b, NO, M0, Stage IIc, and the patient underwent 4 courses of dacarbazine, nimustine, vincristine, and interferon-beta therapy as adjuvant chemotherapy. He had periodic survey for 5 years without recurrence.

He had gastrointestinal endoscopy for medical checkup in 2011, and an elevated tumor with ulcer and brownish punctate distribution was observed in the posterior wall of the lower gastric body (Figure 1). From the upper gastrointestinal examination, a "bull's eye" configuration was observed and gastric metastasis was suspected. The bi- 


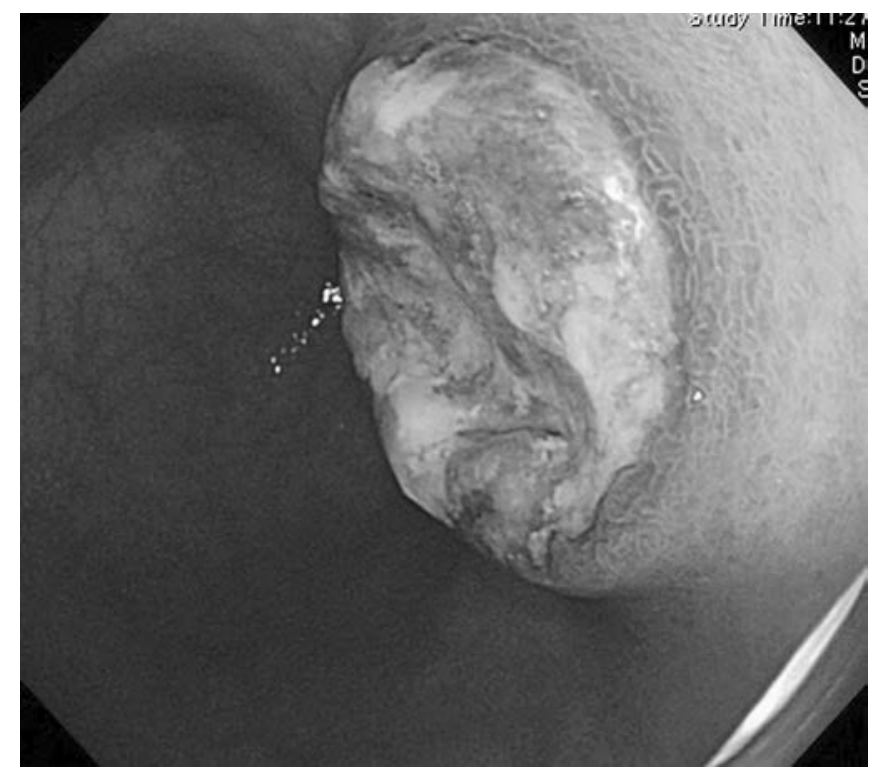

Figure 1. Gastrointestinal endoscopy findings showed elevated tumor with ulcer and brownish punctate distribution in the posterior wall of the lower gastric body.

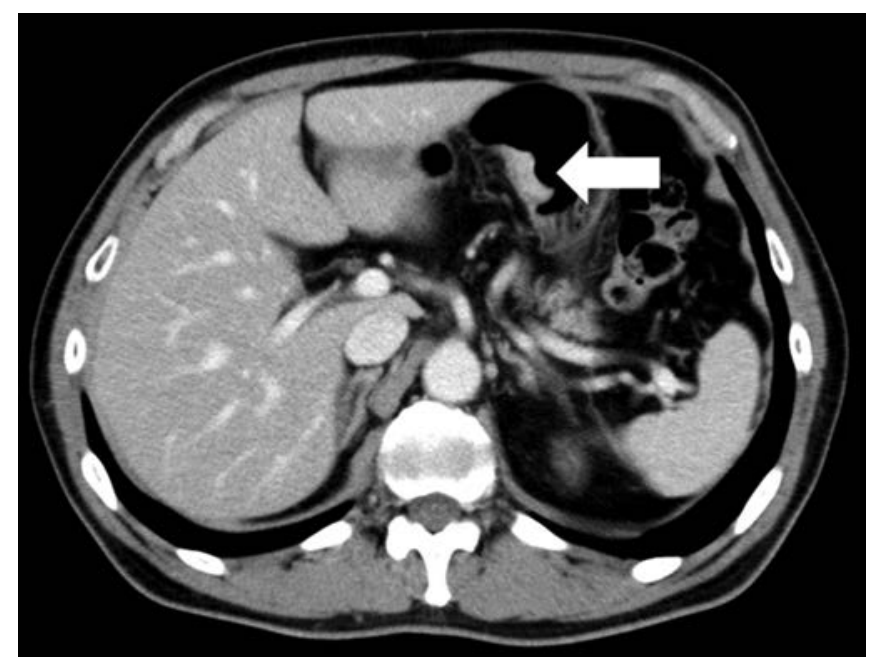

Figure 2. PET/CT scan showed high-intensity spot (white arrow) in the lesser curvature of the stomach. No other highintensity spot was observed. CT, computed tomography; PET, positron-emission tomography.

opsy confirmed the diagnosis as gastric metastasis of the malignant melanoma.

The laboratory test results were within normal limits except for slight elevation of the serum lactic dehydrogenase level $227 \mathrm{IU} / \mathrm{L}$ and the elevation of 5-S-cysteinyldopa $28.5 \mathrm{nmol} / \mathrm{L}$. A contrast computed tomography (CT) scan showed tumor stain in the lesser curvature of
A

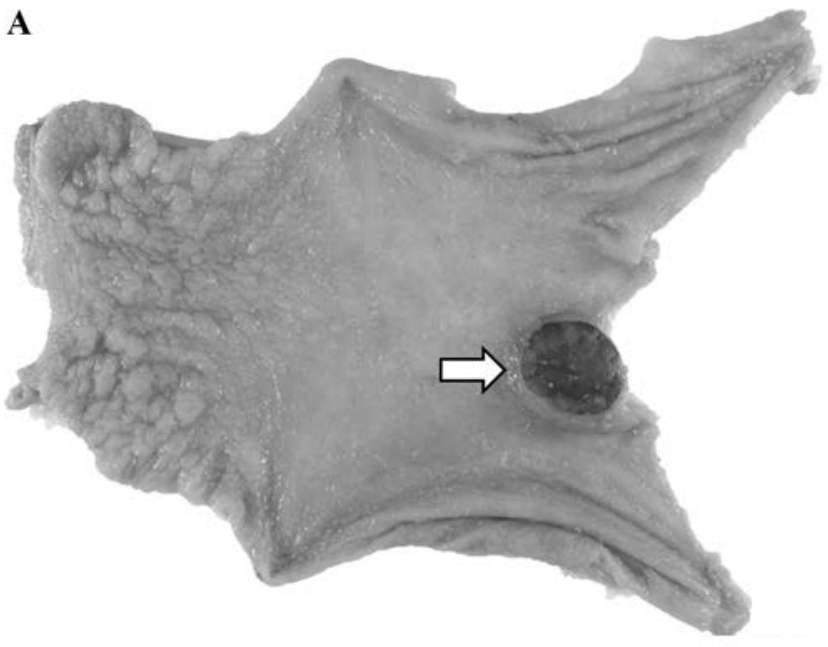

B

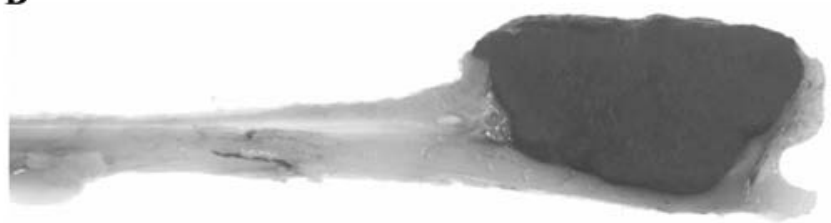

Figure 3. A. The tumor (white arrow) was $2 \mathrm{~cm}$ in diameter with ulceration on the surface. B. The division surface showed sharp marginated tumor.

the stomach. Lymph nodes' swelling and ascites were clinically negative on imaging. Positron-emission tomography (PET)/CT scans were also performed and a high-intensity spot was only seen in the lesser curvature of the stomach (Figure 2).

As there were no other metastases, laparoscopic-assisted distal gastrectomy with lymph node dissection was performed. The black tumor was $2 \mathrm{~cm}$ in diameter and sharply marginated with ulceration on the surface in the resected specimen (Figure 3). Pathological findings of the tumor showed abundance of melanin granule with expansive growth and invasion to the muscularis propria of the stomach (Figures $\mathbf{4}$ and 5 ). The junctional change in the overlying epithelium was not observed. Elastica van Gieson stain and D2-40 stain were performed; however, vascular invasion was beyond determination. Two perigastric lymph nodes metastases, stations no. 3 and no. 7 according to the Japanese Classification of Gastric Carcinoma, 4,5 were also observed in the subcapsular sinus of lymph nodes (Figure 6).

Postoperative course was satisfactory, and he started dacarbazine, nimustine, cisplatin, and tamoxifen therapy. Chest, abdominal, and brain contrast CT and gastrointes- 


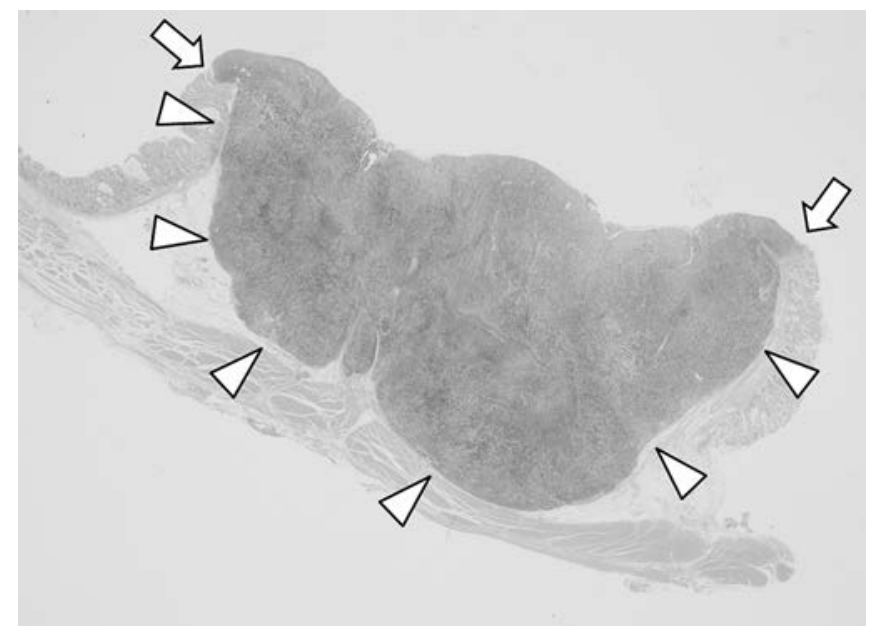

Figure 4. The tumor was microscopically sharply marginated and localized (white arrowheads). No junctional change in the overlying epithelium was observed (white arrow). (Hematoxylin and eosin stain.)

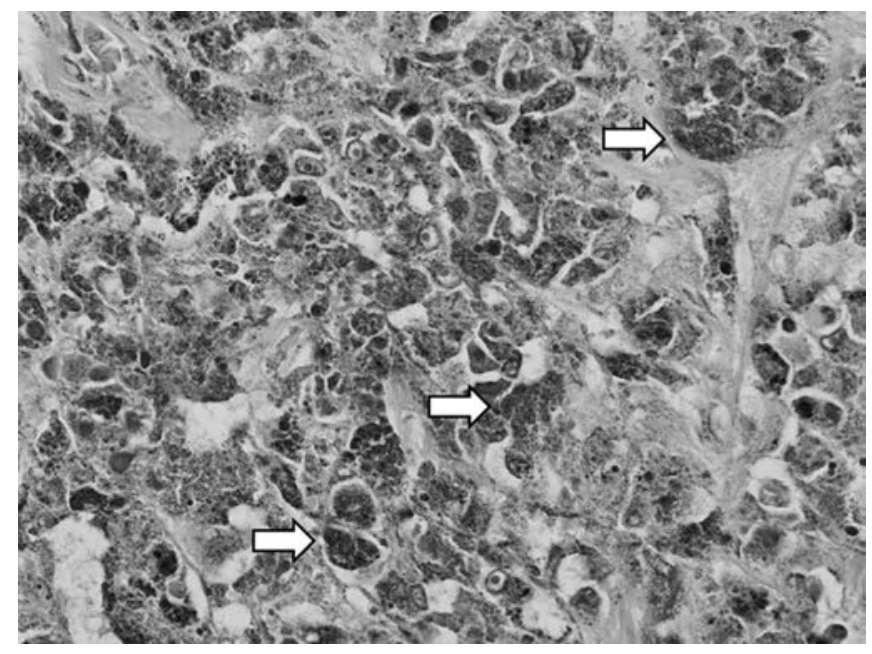

Figure 5. Pathological findings of the tumor showed abundance of melanin granule with expansive growth (white arrows). (Hematoxylin and eosin stain.)

tinal endoscopy were performed every 6 months and PET was performed 1 year after operation. Seventeen months have passed without recurrence.

\section{DISCUSSION}

There have been only few reports of patients with metastatic melanoma in the gastrointestinal tract who underwent surgery. Table $\mathbf{1}^{6-13}$ summarizes the cases with gastrointestinal tract metastases from cutaneous malignant melanoma reported from 2000 to 2012. As reported, the small intestine was the most commonly involved metastatic site. In most

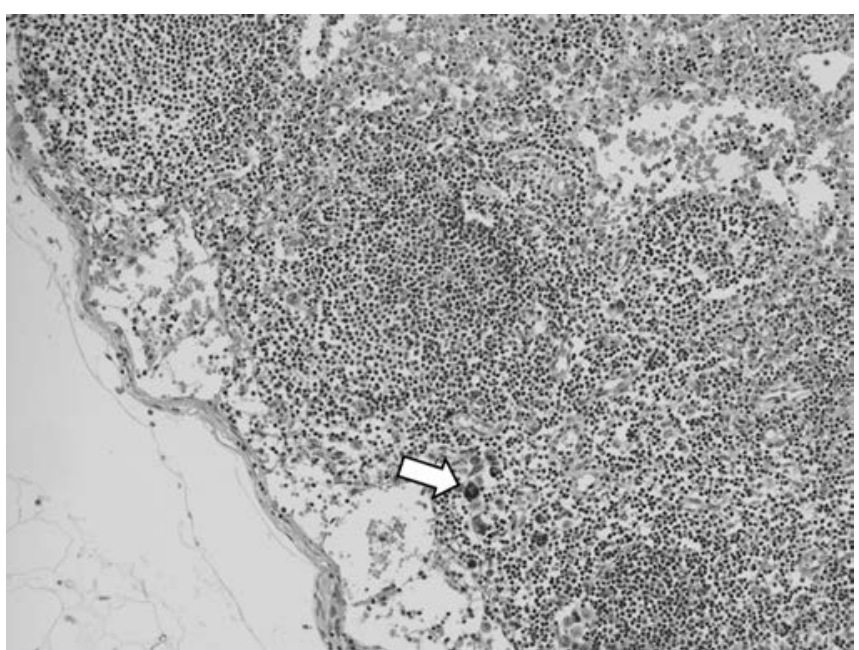

Figure 6. Micrometastases (white arrow) were observed in the subcapsular sinus of lymph nodes. (Hematoxylin and eosin stain.)

cases, detailed examination was performed due to the digestive symptoms, and distant metastases were also detected. Because most cases had multiple distant metastases, reduction therapy were performed to control the digestive symptoms. The cases in which the metastatic site was localized and curative surgical resection was performed had better prognosis than the noncurative surgical resection cases did.

Three types of metastatic malignant melanoma in the gastrointestinal tract are observed by gastrointestinal endoscopy: (1) nodules varying in size, usually appearing to arise on the crest of normal range, often ulcerated at tip, and invariably melanotic; (2) raised submucosal tumor masses with ulcerated centers; (3) mass lesions with varying degrees of necrosis and melanosis. ${ }^{14}$ Types 1 and 2 were also recognized in our case. The difference between these 3 types of the appearance might be the size of the tumor. Enlargement of the tumor might cause the ulceration and necrosis of the center. Histological changes between primary and metastatic malignant melanoma is the absence of junctional change in the overlying epithelium. ${ }^{15}$

The PET scanning for detecting melanoma metastasis had high sensitivity of $84 \%$ to $94 \%$ and specificity of $83 \%$ to $97 \%$, whereas CT showed $55 \%$ to $58 \%$ sensitivity and $70 \%$ to $84 \%$ specificity. ${ }^{16,17}$ PET is the most sensitive method compared with conventional imaging, particularly in soft tissues, lymph nodes, and liver. It also helps to detect sites that are not routinely evaluated by CT. ${ }^{17,18}$ False-negative scans are observed in $4 \%$ of all scans, due to the small size, $<0.3$ to $0.5 \mathrm{~cm}$, or the tumor burden, consisting of only a few cells, that were unable to be resolved by PET scanning. ${ }^{18,19}$ 


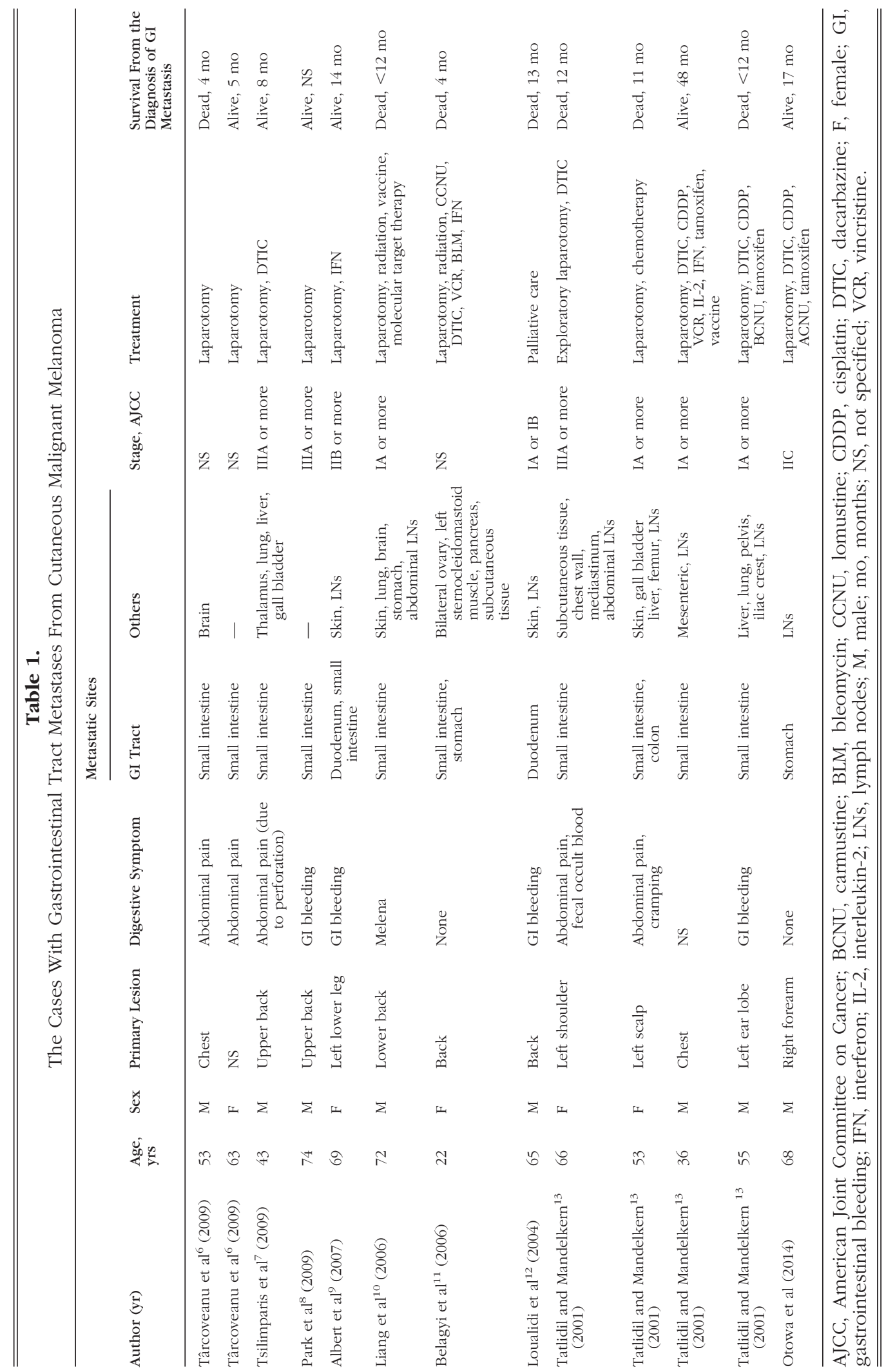


Most patients have distant metastases; however, if the metastasis is localized and resectable, surgical resection should be tried. Some previous studies show longer median survival when curative surgery was performed than for those with nonsurgical treatment. ${ }^{20,21}$

Though chemotherapy is widely used as an alternative treatment, it has been difficult to improve the prognosis. Recently, immunotherapy with ipilimumab has been shown to improve overall survival ${ }^{22}$ and molecular target therapy with vemurafenib has been shown to improve progression free and overall survival in patients with advanced malignant melanoma. ${ }^{23}$ The combined modality using the new treatments might improve the prognosis.

\section{CONCLUSIONS}

In our case, lymph node metastasis was observed in the subcapsular sinus. This might suggest that resection of the metastasis lesion with lymph nodes dissection is recommended for curative surgery even if the PET scan is negative and might lead to a long disease-free interval.

\section{References:}

1. Reintgen DS, Thompson W, Garbutt J, Seigler HF. Radiologic endoscopic and surgical considerations of melanoma metastatic to the gastrointestinal tract. Surgery. 1984;95(6):635-639.

2. Das Gupta TK, Brasfield RD. Metastatic melanoma of the gastrointestinal tract. Arch Surg. 1964;88:969-973.

3. Edge SB, Byrd DR, Compton CC, Fritz AG, Greene FL, Trotti A (Eds). Melanoma of the skin. In: AJCC Cancer Staging Manual. 7th ed. New York, NY: Springer; 2010:325-344.

4. Japanese Gastric Cancer Association. Japanese Classification of Gastric Carcinoma [in Japanese]. 14th ed. Tokyo, Japan: Kanehara; 2010:13.

5. Japanese Gastric Cancer Association. Japanese Classification of Gastric Carcinoma: 3rd English edition. Gastric Cancer. 2011; 14(2):101-112.

6. Târcoveanu E, Dimofte G, Dănilă N, Vasilescu A, Ferariu D, Crumpei F. Small bowel malignant melanoma-report of three cases and review of literature. Acta Chir Belg. 2009;109(6):763-768.

7. Tsilimparis N, Menenakos C, Rogalla P, Braumann C, Hartmann J. Malignant melanoma metastasis as a cause of smallbowel perforation. Onkologie. 2009;32(6):356-358.

8. Park J, Ostrowitz MB, Cohen MS, Al-Kasspooles M. A patient with metastatic melanoma of the small bowel. Oncology (Williston Park). 2009;23(1):98-102.

9. Albert JG, Gimm O, Stock K, Bilkenroth U, Marsch WC, Helmbold P. Small-bowel endoscopy is crucial for diagnosis of melanoma metastases to the small bowel: a case of metachro- nous small-bowel metastases and review of the literature. Melanoma Res. 2007;17(5):335-338.

10. Liang KV, Sanderson SO, Nowakowski GS, Arora AS. Metastatic malignant melanoma of the gastrointestinal tract. Mayo Clin Proc. 2006;81(4):511-516.

11. Belágyi T, Zsoldos P, Makay R, Issekutz A, Oláh A. Multiorgan resection (including the pancreas) for metastasis of cutaneous malignant melanoma. JOP. 2006;7(2):234-240.

12. Loualidi A, Spooren PF, Grubben MJ, Blomjous CE, Goey SH. Duodenal metastasis: an uncommon cause of occult small intestinal bleeding. Neth J Med. 2004;62(6):201-205.

13. Tatlidil R, Mandelkern M. FDG-PET in the detection of gastrointestinal metastases in melanoma. Melanoma Res. 2001; 11(3):297-301.

14. Nelson RS, Lanza F. Malignant melanoma metastatic to the upper gastrointestinal tract: endoscopic and radiologic correlations, form and evolution of lesions, and value of directed biopsy in diagnosis. Gastrointest Endosc. 1978;24(4):156-158.

15. Allen AC, Spitz S. Malignant melanoma; a clinicopathological analysis of the criteria for diagnosis and prognosis. Cancer. 1953;6(1):1-45.

16. Swetter SM, Carroll LA, Johnson DL, Segall GM. Positron emission tomography is superior to computed tomography for metastatic detection in melanoma patients. Ann Surg Oncol. 2002;9(7):646-653.

17. Rinne D, Baum RP, Hör G, Kaufmann R. Primary staging and follow-up of high risk melanoma patients with whole-body $18 \mathrm{~F}$ fluorodeoxyglucose positron emission tomography: results of a prospective study of 100 patients. Cancer. 1998;82(9):1664-1671.

18. Holder WD Jr, White RL Jr, Zuger JH, Easton EJ Jr, Greene FL. Effectiveness of positron emission tomography for the detection of melanoma metastases. Ann Surg. 1998;227(5):764-769.

19. Macfarlane DJ, Sondak V, Johnson T, Wahl RL. Prospective evaluation of 2-[18F]-2-deoxy-D-glucose positron emission tomography in staging of regional lymph nodes in patients with cutaneous malignant melanoma. J Clin Oncol. 1998;16(5):1770-1776.

20. Gutman H, Hess KR, Kokotsakis JA, Ross MI, Guinee VF, Balch CM. Surgery for abdominal metastases of cutaneous melanoma. World J Surg. 2001;25(6):750-758.

21. Agrawal S, Yao TJ, Coit DG. Surgery for melanoma metastatic to the gastrointestinal tract. Ann Surg Oncol. 1999;6(4):336-344.

22. Hodi FS, O’Day SJ, McDermott DF, et al. Improved survival with ipilimumab in patients with metastatic melanoma. $N$ Engl J Med. 2010;363(8):711-723.

23. Chapman PB, Hauschild A, Robert C, et al., for the BRIM-3 Study Group. Improved survival with vemurafenib in melanoma with BRAF V600E mutation. N Engl J Med. 2011;364(26):2507-2516. 